infectious) than the initial disease from the first encounter with the virus ${ }^{10,11}$. All these observations support the proposal of careful clinical observation of the disease that may eventually affect vaccinated patients, including monitoring selected at-risk people for asymptomatic infection and infectivity. Ultimately, the most information about immune escape is learned in the clinic, when there is a variant-specific drop-off of efficacy, along with a propensity for reinfection as a function of particular variants. The in vitro assays are expected to correlate with the clinical findings, as surrogate metrics to indicate what will be expected to manifest in patients.

Finally, should SARS-CoV-2 be able to perfectly escape vaccine-induced immunity, rapid modification of mRNA vaccines could provide an immunity boost likely to provide protection against the new viral strains as well. However, it is important to be aware of the existence of and the sequence of such variants in the shortest possible time frame in order to mitigate risk and control the consequences.

The Italian word for concern is 'preoccupazione', which comes from 'occupazione' ('take care') and 'pre' ('in advance'). Simply put, this is what needs to be done. Concern about SARS-CoV-2 variants should not cause panic but instead should prompt efforts to ensure that all the necessary steps for mitigating variant impact are taken in advance through the development of tools that detect and characterize these new strains in a timely and standard manner.
Published online: 1 March 2021

https://doi.org/10.1038/s41591-021-01290-0

References

1. European Centre for Disease Prevention and Control. https:// www.ecdc.europa.eu/sites/default/files/documents/SARS-CoV2-variant-multiple-spike-protein-mutations-United-Kingdom.pdf (20 December 2020).

2. Korber, B. et al. Cell 182, 812-827.e19 (2020).

3. Burton, D. R. Nat. Rev. Immunol. 2, 706-713 (2002).

4. Strebel, P. M. \& Orenstein, W. A. N. Engl. J. Med. 381, 349-357 (2019)

5. Lambert, N., Strebel, P., Orenstein, W., Icenogle, J. \& Poland, G. A. Lancet 385, 2297-2307 (2015).

6. Karlsson Hedestam, G. B. et al. Nat. Rev. Microbiol. 6, 143-155 (2008)

7. Eriksen, J. et al. Infect. Dis. 53, 1-8 (2021).

8. Qiangian, L. et al. Rev. Med. Virol. 28, e1963 (2018).

9. Tuaillon, E. et al. J. Immunol. Methods 315, 144-152 (2006).

10. Henderson, F. W., Collier, A. M., Clyde, W. A. Jr. \& Denny, F. W. N. Engl. J. Med. 300, 530-534 (1979).

11. Glezen, W. P., Taber, L. H., Frank, A. L. \& Kasel, J. A. Am. J. Dis. Child. 140, 543-546 (1986).

\title{
The need for ethical guidance for the use of patient-reported outcomes in research and clinical practice
}

To the Editor-Patient-reported outcomes (PROs) are increasingly being used in clinical research to provide evidence of the benefits and risk of therapy from a patient perspective. PRO data from clinical trials can inform regulatory approvals and drug labeling, clinical guideline development and health policy ${ }^{1}$. Approximately one third of clinical trials include PROs collected through the use of patient questionnaires $^{2}$. Beyond trials, PRO data are also increasingly captured in observational research and routine clinical care to provide information on the burden of disease and real-world evidence of treatment safety and effectiveness ${ }^{3}$, for audit and benchmarking ${ }^{1}$, and to monitor the status of patients and provide timely care tailored to individual needs. For instance, a study demonstrated that systematic web-based collection of information on symptoms led to improved health-related quality of life, survival and quality-adjusted survival, and fewer visits to the emergency room and hospitalization, among patients receiving chemotherapy for advanced solid tumors ${ }^{4}$. Patients value PRO trial results, as they can enhance clinicianpatient communication about treatment options, which helps patients to feel more empowered in shared decision-making around their care ${ }^{5}$.

Despite the benefits of incorporating PROs in research and routine practice, several ethical challenges can hinder the uptake and benefit to patients of PRO data. The PRO content of trial protocols and reporting of $\mathrm{PRO}$ results are often suboptimal, missing data rates are high, and delay of the publication of PRO data is commonplace. A recent study evaluating 228 studies from the National Institute of Health Research Cancer Portfolio demonstrated that 50,000 patients were involved in studies that failed to publish the PRO data collected, which is considered unethical ${ }^{6}$.

PRO data collection is associated with a number of ethical considerations that must be addressed. An ethical consideration is defined as one that requires a choice based on moral considerations drawing on established principles, theories and values, that might have implications for the person's or society's welfare. The differing use of PROs in research and routine care settings, and review and/or use of data by clinical teams, may lead to uncertainties for patients about why data are being collected and data privacy-how their data are being viewed and used. Research indicates that in some instances, PRO measures may not reflect the perspectives of vulnerable groups or older people, which challenges bioethical principles and threatens the scientific validity of results ${ }^{7}$. The burden on patients associated with the completion of multiple questionnaires is also a concern. Of particular note is the lack of guidance on how staff should manage situations in which PRO data reveal 'concerning' levels of psychological distress or physical symptoms that may require an immediate response . $^{8}$. Evidence suggests research staff are handling such data inconsistently, which may lead to inequitable patient care, co-intervention bias and confusion.

Furthermore, PROs could be used for long-term follow-up to assess the impact of the coronavirus SARS-CoV-2 on patients' quality of life and alert clinicians of potential life-threating symptoms ${ }^{9}$. The increased use of telehealth will also heighten the use of PRO data to monitor patients' symptoms. Therefore, there is a need to ensure that this is done in an ethical way that protects patients' safety and data.

To address these challenges, the PRO Ethics Steering Group, composed of PRO methodologists, patient partners 
and ethicists, is developing international, consensus-based guidelines for use by researchers and patient partners in preparing ethics submissions and for use by research ethics committees and institutional review boards in the assessment of PRO research. The guidelines will focus specifically on ethical considerations of PRO research and data collection in clinical practice, using methodological guideline development of the EQUATOR (Enhancing Quality and Transparency of Health Research) Network ${ }^{10}$. The development process will include a literature review, a modified Delphi exercise and an international consensus meeting involving members of research ethics committees, experts in research ethics, patient partners, trialists and PRO researchers. Given the dearth of guidance currently available, the authors plan to hold the Delphi exercise and consensus meeting with a view to publishing the guideline in 2021 .

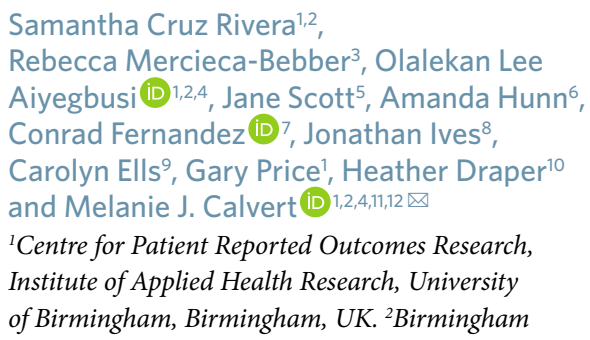

Health Partners Centre for Regulatory Science and Innovation, University of Birmingham, Birmingham, UK. ${ }^{3}$ Faculty of Medicine, Sydney Medical School, Central Clinical School, University of Sydney, Sydney, New South Wales, Australia. ${ }^{4}$ National Institute for Health Research Applied Research Centre West Midlands, Birmingham, UK. ${ }^{5} J a n s s e n$ Global Services, Johnson \& Johnson, High Wycombe, UK. ${ }^{6}$ A J Hunn Associates, London, UK. ${ }^{7}$ Division of Haematology-Oncology, IWK Health Care Centre, Halifax, Nova Scotia, Canada. ${ }^{8}$ Bristol Medical School, Bristol Population Health Science Institute, University of Bristol, Bristol, UK. ${ }^{9}$ School of Population and Global Health, McGill University, Montreal, Quebec, Canada. ${ }^{10}$ Social Science and Systems in Health, University of Warwick, Warwick, UK. ${ }^{11}$ NIHR Birmingham Biomedical Research Centre, NIHR Surgical Reconstruction and Microbiology Research Centre, University of Birmingham, Birmingham, UK. ${ }^{12}$ Health Data Research UK, London, UK.

$凶_{e-m a i l: m . c a l v e r t @ b h a m . a c . u k}$

Published online: 4 March 2021

https://doi.org/10.1038/s41591-021-01275-Z

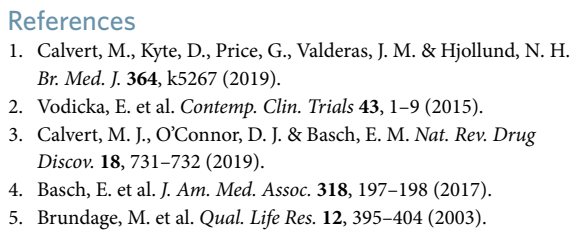

6. Kyte, D. et al. J. Natl. Cancer Inst. Monogr. 111, 1170-1178 (2019).

7. Hagell, P., Reimer, J. \& Nyberg, P. Value Health 12, 613-617 (2009).

8. Kyte, D., Draper, H. \& Calvert, M. J. Am. Med. Assoc. 310, 1229-1230 (2013).

9. Aiyegbusi, O. L. \& Calvert, M. J. Lancet 396, 531-531 (2020).

10. Moher, D., Schulz, K. F., Simera, I. \& Altman, D. G. PLoS Med. 7, e1000217 (2010).

\section{Acknowledgements}

M.J.C. receives funding from the National Institute for Health Research (NIHR) Birmingham Biomedical Research Centre, the NIHR Surgical Reconstruction and Microbiology Research Centre and NIHR ARC West Midlands at the University of Birmingham and University Hospitals Birmingham NHS Foundation Trust, Health Data Research UK, Innovate UK (part of UK Research and Innovation). The views expressed in this article are those of the author(s) and not necessarily those of the NIHR, or the Department of Health and Social Care. R.M.B. is supported by the Australian Government by a National Health and Medical Research Early career fellowship.

\section{Author contributions}

S.C.R. and M.J.C. conceived of the idea; S.C.R. developed the first draft; and all authors made substantial revisions and approved the final manuscript.

\section{Competing interests}

M.J.C. receives funding from Macmillan Cancer Support and UCB Pharma, and has received personal fees from Astellas, Takeda, Merck, Daiichi Sankyo Glaukos, GlaxoSmithKline and the Patient-Centered Outcomes Research Institute outside the submitted work. O.L.A. declares personal fees from Gilead Sciences and GlaxoSmithKline outside the submitted work. J.S. is an employee of Janssen-Cilag UK and holds stock in Johnson \& Johnson.

\section{Do not sell regulatory science short}

To the Editor-A recent federal notice proposes to permanently remove oversight by the US Food and Drug Administration (FDA) over 91 medical devices, including several devices that apply artificial intelligence and those under temporary COVID-19 waiver. Public needs during a response to an unprecedented public-health emergency aside, the federal notice sends an alarming message - that regulatory science and applying its principles during regulatory review are unnecessary.

The evidence for removal is the lack of adverse events reported in an FDA database ${ }^{2}$. Assuming that all relevant adverse events are accurately identified in the 'real world', and further assuming that all of those adverse events are accurately reported in the FDA database, then the absence of adverse events would indicate that the screened devices are safe and are of 'low risk'. However, even with those arguably unproven assumptions, the cause for the absence of adverse events has many attributable factors, including the FDA review process itself, which is now subject to removal - simply put, the safety of the current devices driving the removal might be in part attributable to those devices having undergone independent, scientifically sound FDA review. The proposed permanent changes thereby represent a manifestation of the principle that 'nobody ever gets credit for fixing problems that never happened'3 ${ }^{3}$; however, it is exactly in this context that regulatory science, with its diverse tools, standards and approaches, is necessary to ensure safety, efficacy, quality and performance to help prevent adverse events ${ }^{4}$.

After decades of public funding and numerous strategic governmental initiatives ${ }^{5}$, regulatory science today is a firmly established hard science recognized and championed by the FDA. Regulatory science is, however, not restricted to the
FDA-numerous scientists contribute continuously via methods, tools and standards to facilitate and inform regulatory decision-making. The partnership between science and the 'regulatory ecosystem' have brought stakeholders together ${ }^{6}$ to begin to tackle very difficult problems, including how to regulate continuously learning artificial-intelligence tools or generative adversarial networks. There are numerous unanswered questions that represent an opportunity for all stakeholders to come together and drive development toward a comprehensive and agile regulatory framework ${ }^{7}$. In other words, the federal notice highlights the exact purposes for which regulatory science existsnamely, that rigorous review of passive event-reporting systems and long-term monitoring to elucidate causal relationships of adverse events is necessary.

Accelerating medical innovation requires the collaborative generation of logically 